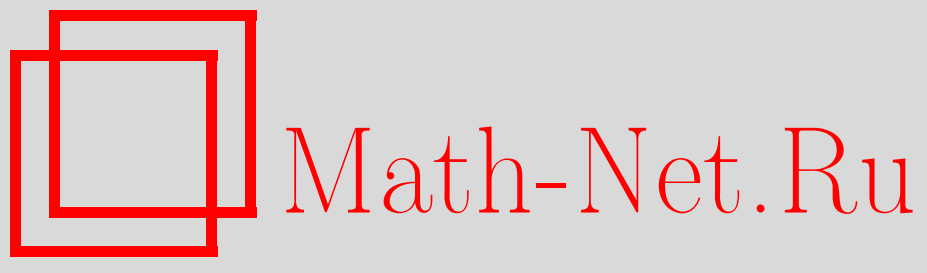

О. В. Шварцман, Свободные алгебры автоморфных форм на верхней полуплоскости, Функи. анализ и его прил., 2003, том 37, выпуск 2, 81-89

DOI: https://doi.org/10.4213/faa151

Использование Общероссийского математического портала Math-Net.Ru подразумевает, что вы прочитали и согласны с пользовательским соглашением

http: //www . mathnet.ru/rus/agreement

Параметры загрузки:

IP : 35.173 .219 .12

26 апреля 2023 г., 16:10:10

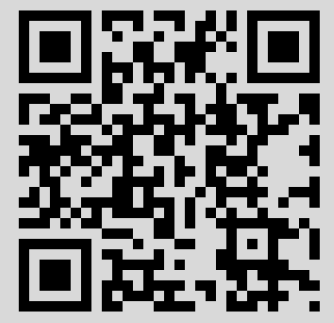


2003, т. 37, вып. 2, с. 81-89

УДК $515.173+512.745$

\title{
Свободные алгебры автоморфных форм на верхней полуплоскости*
}

\author{
(C) 2003. О. В. ШВАРЦМАН
}

В работе найдены все пары $(\Gamma, a)$, состоящие из кокомпактной фуксовой группы $\Gamma$ рода нуль и ее фактора автоморфности $a$, для которых градуированная алгебра $a$-Г-автоморфных форм свободна.

\section{§1. Коциклы}

Пусть $\Gamma$ - дискретная группа автоморфизмов верхней полуплоскости $H$, такая, что факторпространство $X=H / \Gamma$ компактно. Функция $a(\gamma, z)$ двух переменных $\gamma \in \Gamma, z \in H$ называется коциклом (или фактором автоморфности) группы $Г$, если она

(a) при фиксированном $\gamma$ голоморфна по $z$ и нигде на $H$ не обращается в нуль,

(b) удовлетворяет условию

$$
a\left(\gamma_{1} \gamma_{2}, z\right)=a\left(\gamma_{1}, \gamma_{2} z\right) a\left(\gamma_{2}, z\right) .
$$

По каждой голоморфной нигде не равной нулю функции $f(z)$ строится тривиальный коцикл $e(\gamma, z)=f(\gamma z) / f(z)$.

Два коцикла группы Г называются эквивалентными, если один из них получается из другого умножением на тривиальный коцикл.

Классы эквивалентных коциклов образуют абелеву группу по умножению, об устройстве которой (в интересующем нас случае) будет сказано ниже.

ПримеР. Положим $j(\gamma, z)=\left.\frac{d}{d z}(\gamma z)\right|_{z}$. Условие (1) выполняется в силу цепного правила дифференцирования. Коциклы вида $a=j^{n}, n \in \mathbb{Z}$, называются классическими.

С помощью коцикла $a(\gamma, z)$ определим действие группы $Г$ в пространстве $L=H \times \mathbb{C}:$

$$
\gamma(z, t)=(\gamma z, a(\gamma, z) t), \quad z \in H, t \in \mathbb{C}, \gamma \in \Gamma .
$$

При этом тривиальное линейное голоморфное расслоение $L$ над $H$ превращается в (вообще говоря) нетривиальное Г-расслоение $L(a)$. Эквивалентные коциклы определяют изоморфные Г-расслоения. На языке Г-расслоений основной результат статьи в меньшей общности был анонсирован в [4].

\section{§2. Алгебра автоморфных форм $A=A(\Gamma, a)$. Цель работы}

Голоморфная в $H$ функция $f(z)$ называется $a$-Г-автоморфной формой (чаще просто формой) целого веса $l$, если она удовлетворяет функциональному уравнению

$$
f(\gamma z)=a^{l}(\gamma, z) f(z) .
$$

*Работа поддержана грантом DFB-РФФИ 99-01-04024. 
Через $A_{l}$ обозначим $\mathbb{C}$-линейное пространство таких форм и рассмотрим градуированную алгебру

$$
A=A(\Gamma, a)=\bigoplus_{l \in \mathbb{Z}} A_{l} .
$$

Цель статьи - указать все пары $(\Gamma, a)$, для которых алгебра $A(\Gamma, a)$ свободна, т. е. изоморфна градуированной алгебре многочленов от двух переменных $\mathbb{C}[X, Y]$ (теорема в $\$ 4)$.

ЗАмЕчАНИЕ. В 80-е годы алгебры $A(\Gamma, a)$ интенсивно изучались рядом авторов в связи с теорией В. И. Арнольда квазиоднородных двумерных особенностей в рамках программы исследований, инициированной статьей И. В. Долгачева [2].

Но вопрос о том, когда алгебра $A(\Gamma, a)$ является свободной, важный с точки зрения теории инвариантов, этими авторами не ставился по понятной причине: тот факт, что алгебра $A$ свободна, означает, что рассматриваемая точка как раз неособа. Отметим также, что в большинстве работ (например, в [13]) рассматриваются только классические алгебры $A\left(\Gamma, j^{-1}\right)$, что резко обедняет ситуацию: алгебры $A\left(\Gamma, j^{-1}\right)$ никогда не бывают свободными для кокомпактных фуксовых групп и редко бывают свободными для фуксовых решеток (например, для модулярной группы эта алгебра свободна).

\section{§3. Предварительные замечания и известные факты}

3.1. Роль комплексных отражений. Рассмотрим действие группы $\Gamma$ в расслоении $L(a)$ (см. формулу $(2))$. Говорят, что элемент конечного порядка $\gamma \in \Gamma$ действует в $L(a)$ как комплексное отражение, если множество его неподвижных точек в $L(a)$ связно и имеет комплексную коразмерность 1 . Какие элементы из $\Gamma$ действуют в $L(a)$ как комплексные отражения? Нетрудно понять, что комплексное отражение в $L(a)$ - это в точности эллиптический элемент $\gamma \in \Gamma$, тождественно действующий в слое над своей единственной неподвижной точкой $z(\gamma) \in H$, что эквивалентно выполнению условия $a(\gamma, z(\gamma))=1$.

В [5] установлен важный для нас

ФАКТ 1. Если алгебра $A(\Gamma, a)$ свободна, то группа $\Gamma$, действующия в $L(a)$, порождается комплексными отражениями.

Отсюда, в частности, следует, что фуксова группа $Г$ порождается эллиптическими элементами, т.е. является фуксовой группой рода 0 с сигнатурой $\left(0 ; n_{1}, \ldots, n_{r}\right)$.

3.2. Фуксова группа рода нуль и структура ее группы классов коциклов. Зафиксируем фуксову группу $\Gamma\left(0 ; n_{1}, \ldots, n_{r}\right)$ рода 0 . По определению $H / \Gamma \simeq$ $\mathbb{P}^{1}(\mathbb{C})$. Договоримся всюду в дальнейшем через $\bar{x}$ обозначать проекцию точки $x \in H$ на факторпространство $\mathbb{P}^{1}(\mathbb{C})$.

Хорошо известно [7], что группа $Г$ допускает каноническую систему образующих $\gamma_{1}, \ldots, \gamma_{r}$ с определяющими соотношениями $\gamma_{i}^{n_{i}}=1, i=1, \ldots, r$, $\gamma_{1} \ldots \gamma_{r}=1$ (не следует думать, что эти образующие совпадают с теми, о которых идет речь в формулировке факта 1). Любой эллиптический элемент из группы $Г$ сопряжен степени ровно одной из образующих $\gamma_{i}$. В частности, любая точка $x \in H$ с нетривиальным стабилизатором $\Gamma_{x}$ эквивалентна ровно одной из точек $P_{i}=z\left(\gamma_{i}\right)$.

В окрестности неподвижной точки $z\left(\gamma_{i}\right)=P_{i}$ можно выбрать локальный параметр $w$ так, что действие $\gamma_{i}$ в этой окрестности будет линейным: $\gamma_{i}(w)=$ 
$e^{2 \pi \sqrt{-1} / n_{i}} w$. Условие (1) показывает, что $a\left(\gamma_{i}, P_{i}\right)=e^{2 \pi \sqrt{-1} d_{i} / n_{i}}, 0 \leqslant d_{i}<n_{i}$, $i=1, \ldots, r$. Опишем группу классов коциклов группы $\Gamma$. С этой целью рассмотрим множество $C$ всех векторов вида $\left(x_{0}, x_{1}, \ldots, x_{r}\right)$, где $x_{i} \in \mathbb{Z}$ и $0 \leqslant x_{j}<n_{j}$, $j=1, \ldots, r$. Складывать такие векторы мы будем покоординатно, соблюдая такое правило: $k$-е координаты при $k>0$ складываются по модулю $n_{k}$, и если происходит «переполнение», то к координате с номером 0 результата прибавляется единица.

Множество $C$ с такой операцией сложения является группой.

ФАкт 2. Группа классов коциклов группь $\Gamma\left(0 ; n_{1}, \ldots, n_{r}\right)$ изоморфна группе С. Изоморфизм осуществляется отображением, которое классу коцикла а ставит в соответствие вектор $C(a)=\left(\mathrm{Ch}(a), d_{1}, \ldots, d_{r}\right)$, где $\mathrm{Ch}(a)-$ число Черна Г-расслоения $L(a)$, a $a\left(\gamma_{i}, P_{i}\right)=e^{2 \pi \sqrt{-1} d_{i} / n_{i}}$ (cм., например, [9] или [10]).

Будем называть вектор $C(a)$ кодом коцикла $a$.

Пример. Канонический коцикл $j^{-1}$ для треугольной группы $\Gamma=T(2,3,7)$ кодируется вектором $(-2 ; 1,2,6)$. Код коцикла $j^{-2}$ равен $(-1 ; 0,1,5)$.

3.3. Формула Римана-Роха для коциклов фуксовых групп рода 0 и размерность пространства автоморфных форм. Обозначим через $h(\Gamma, a)$ размерность пространства голоморфных $a$-Г-автоморфных форм.

ФАКТ $3[1,3]$. Имеет место равенство

$$
h(\Gamma, a)-h\left(\Gamma, a^{-1} \cdot j^{-1}\right)=1+\mathrm{Ch}(a) .
$$

Из формулы (4) уже несложно получить формулу для $h(\Gamma, a)$.

ЛЕмма 1. Eсли $h(\Gamma, a) \neq 0$, mo $h\left(\Gamma, a^{-1} \cdot j^{-1}\right)=0$, u наоборот.

ДокАЗАТЕЛьство. Пусть $f-$ ненулевая $a$-Г-автоморфная форма, а $g-$ ненулевая $a^{-1} \cdot j^{-1}$-Г-автоморфная форма. Тогда $f \cdot g-$ ненулевая $j^{-1}-\Gamma$-автоморфная форма, представляющая ненулевой голоморфный дифференциал на факторпространстве $H / \Gamma=P^{1}(\mathbb{C})$. Противоречие.

Как следствие этой леммы и фактов 2 и 3 получаем формулу для размерности $l$-й градуированной компоненты $A_{l}$ алгебры $A(\Gamma, a)$ при условии, что эта компонента не нульмерна:

$$
\operatorname{dim} A_{l}=1+l \operatorname{Ch}(a)+\sum_{i=1}^{r}\left[\frac{l d_{i}}{n_{i}}\right]
$$

для коцикла $а$ с кодом $C(a)=\left(\mathrm{Ch}(a), d_{1}, \ldots, d_{r}\right)$.

ЗАмЕчАНИЕ. Для коцикла $a=j^{-1}$ это формула Шимуры из книги [6]. Данное там доказательство легко переносится на общий случай.

Полезным окажется и такое прямое следствие факта 3.

ЛЕмма 2. Если $1+\mathrm{Ch}(a) \geqslant 0, \operatorname{mo} h(\Gamma, a)=1+\mathrm{Ch}(a)$.

3.4. Формула для подсчета нулей. Для любой (даже мероморфной) $a$-Г-автоморфной формы $f$ можно говорить о кратности $O(\bar{x})$ ее нуля (полюс - нуль отрицательной кратности) в любой точке $\bar{x} \in P^{1}(\mathbb{C})$, подразумевая под этим кратность, с которой она обращается в нуль в любой точке орбиты $\{\Gamma x\} \subset H$.

Прежде всего, элементарное вычисление показывает, что для коцикла $a$ с кодом $C(a)=\left(\operatorname{Ch}(a), d_{1}, \ldots, d_{r}\right)$ имеет место сравнение $O\left(\bar{P}_{i}\right) \equiv d_{i}\left(\bmod n_{i}\right)$ 
(напомним, что $\left.\gamma_{i}\left(P_{i}\right)=P_{i}\right)$. Следующий факт для классических коциклов был известен еще Клейну и Пуанкаре.

ФАКт 4 [3]. Если $f$ - ненулевая а-Г-автоморфная форма веса $l$, то

$$
\sum \frac{O(\bar{x})}{\left|\Gamma_{\bar{x}}\right|}=l\left(\mathrm{Ch}(a)+\sum_{1}^{r} \frac{d_{i}}{n_{i}}\right)
$$

(суммирование в левой части формуль ведется по всем точкам факторпространства $\left.P^{1}(\mathbb{C})\right)$.

3.5. Остается зафиксировать несколько обозначений: $p^{\prime} / q^{\prime}$ будет обозначать несократимую запись дроби $p / q,(a, b)$ - наибольший общий делитель, а $[a, b]$ - наименьшее общее кратное чисел $a$ и $b$.

\section{§4. Основной результат}

Пусть $\Gamma$ - фуксова группа сигнатуры $\left(0 ; n_{1}, \ldots, n_{r}\right)$ и $a(\gamma, z)-$ ее коцикл с кодом $C(a)=\left(\mathrm{Ch}(a), d_{1}, \ldots, d_{r}\right)$.

ТЕоремА. Для того итобы алгебра $A(\Gamma, a)$ была свободна, необходимо $u$ достаточно, чтобы а был коциклом одного из трех перечисленных ниже взаимоисключающих типов.

0) $C(a)=(1 ; 0, \ldots, 0)$.

$B$ этом случае $A=\mathbb{C}[\varphi, \psi], \operatorname{deg} \varphi=\operatorname{deg} \psi=1$ и формы $\varphi$ и $\psi$ можно выбрать так, чтобы $\varphi$ имела единственный простой нуль в произвольной точке $\bar{x} \in P^{1}-\left\{\bar{P}_{1}, \ldots, \bar{P}_{r}\right\}$, а $\psi$ - единственный простой нуль в произвольной точке $\bar{y} \in P^{1}-\left\{\bar{P}_{1}, \ldots, \bar{P}_{r}\right\}$, отличной от точки $\bar{x}$.

1) $C(a)=\left(0 ; 0, \ldots, d_{i}, \ldots, 0\right)\left(d_{i} \neq 0\right.$ ровно для одного индекса $\left.i\right)$ и выполняется условие $d_{i} / n_{i}=1 / n_{i}^{\prime}$ (m.e. $d_{i}$ делит $\left.n_{i}\right)$.

В этом случае $A=\mathbb{C}[\varphi, \psi], \operatorname{deg} \varphi=1, \operatorname{deg} \psi=n_{i}^{\prime}$, форма $\varphi$ имеет единственный нуль кратности $d_{i}$ в точке $\bar{P}_{i}$, а $\psi$ можно выбрать так, чтобы она имела единственный простой нуль в произвольной точке $\bar{x} \in P^{1}-$ $\left\{\bar{P}_{1}, \ldots, \bar{P}_{r}\right\}$.

2) $C(a)=\left(-1 ; 0, \ldots, d_{i}, \ldots, d_{j}, \ldots, 0\right)\left(d_{i} \neq 0 u d_{j} \neq 0\right.$ ровно для двух индексов $i$ и $j)$ и выполнено условие

$$
\frac{d_{i}^{\prime}}{n_{i}^{\prime}}+\frac{d_{j}^{\prime}}{n_{j}^{\prime}}=1+\frac{1}{n_{i}^{\prime} n_{j}^{\prime}}
$$

В этом случае $A=\mathbb{C}[\varphi, \psi], \operatorname{deg} \varphi=n_{i}^{\prime}, \operatorname{deg} \psi=n_{j}^{\prime}$, форма $\varphi$ имеет единственныци нуль кратности $\left(d_{j}, n_{j}\right)$ в точке $\bar{P}_{j}$, а форма $\psi$ - единственный нуль кратности $\left(d_{i}, n_{i}\right)$ в точке $\bar{P}_{i}$.

\section{§5. Доказательство необходимости части условий теоремы}

5.1. Обозначим через $I$ множество индексов $j$, для которых $d_{j} \neq 0$.

ПреДЛОЖЕНИЕ 1. Если алгебра $A=A(\Gamma, a)$ свободна, mо $|I| \leqslant 2$.

ДокАЗАтЕЛьство. Рассмотрим действие группы $Г$ в расслоении $L(a)$, и пусть $\Gamma^{\prime}$ - нормальная подгруппа, порожденная всеми комплексными отражениями из группы $Г$. Согласно факту 1 , группа $\Gamma^{\prime}$ совпадает с $\Gamma$. С другой стороны, любое отражение из Г сопряжено отражению, принадлежащему одной из подгрупп 
$\Gamma_{P_{i}}^{\prime}$ (см. пп. 3.1 и 3.2; значок «штрих» для подгруппы имеет тот же смысл, что и для всей группы). Следовательно, группа $\Gamma^{\prime}$ нормально порождается своими подгруппами $\Gamma_{P_{i}}^{\prime}$. Легко видеть, что $\Gamma_{P_{i}}^{\prime}=\left\langle\gamma_{i}^{q_{i}}\right\rangle$, где $q_{i}=n_{i} /\left(n_{i}, d_{i}\right)$, и копредставление группы $\Gamma$ (п. 3.2) позволяет сделать вывод, что факторгруппа $\Gamma / \Gamma^{\prime}$ порождается элементами $\tilde{\gamma}_{i}, i \in I$, с определяющими соотношениями $\tilde{\gamma}_{i}^{q_{i}}=1$, $i \in I, \prod_{j \in I} \tilde{\gamma}_{j}=1$. А про такую группу известно [7], что тривиальна она бывает, только если $|I|=0,1,2$.

Итак, предложение 1 разбивает все интересные нам коциклы на три типа нулевой, первый и второй, согласно числу отличных от нуля $d_{i}$ в коде коцикла. Теперь мы покажем, что числа Черна у коциклов каждого из этих типов обязательно такие, как в теореме. Но сначала дадим одно полезное определение.

ОПрЕДЕЛЕНИЕ. Ненулевая автоморфная форма веса $l$ называется одинокой, если пространство всех форм веса $l$ одномерно.

ПреДЛОЖЕНИЕ 2. Если алгебра $A(\Gamma, a)$ свободна, mо $\mathrm{Ch}(a)=1,0$ или -1 для коциклов нулевого, первого и второго типов (соответственно).

ДокАЗАТЕЛьство. Пусть $A=\mathbb{C}[\varphi, \psi]$, причем $m=\operatorname{deg} \varphi \leqslant \operatorname{deg} \psi$.

Случай коцикла нулевого типа. По формуле (5) получаем $\operatorname{dim} A_{m}=m \operatorname{Ch}(a)$ +1 . Но так как левая часть равенства положительна и не превосходит двух, то число Черна может принимать только два значения: 0 или 1 . Случай $\operatorname{Ch}(a)=0$ невозможен, так как при этом размерность любой ненулевой однородной компоненты нашей алгебры равна 1, что, конечно, не так. Следовательно, $\mathrm{Ch}(a)=1$. Более того, как показывает формула для размерности, алгебра $A$ порождается двумя формами веса 1 .

Случай коциикла первого типа (можно считать, что $d_{1} \neq 0$ ). На этот раз с помощью формулы (5) получаем, что $0<\operatorname{dim} A_{m}=1+m \operatorname{Ch}(a)+\left[m d_{1} / n_{1}\right] \leqslant 2$. Поскольку $\left[m d_{1} / n_{1}\right] \leqslant m-1$, то число Черна может принимать только два значения: 0 или 1. При этом, если $\operatorname{Ch}(a)=1$, то обязательно $m=1$ и $\operatorname{deg} \varphi=\operatorname{deg} \psi=1$. Но тогда $\operatorname{dim} A_{n_{1}}=1+n_{1}$. С другой стороны, формула для размерности в этом случае дает $\operatorname{dim} A_{n_{1}}=1+n_{1}+d_{1}>1+n_{1}$. Противоречие. Остается единственная возможность $\mathrm{Ch}(a)=0$.

Случай коциикла второго типа (можно считать, что $d_{1}$ и $d_{2}$ не равны 0 ). Исходное неравенство принимает вид

$$
0<\operatorname{dim} A_{m}=1+m \operatorname{Ch}(a)+\left[\frac{m d_{1}^{\prime}}{n_{1}^{\prime}}\right]+\left[\frac{m d_{2}^{\prime}}{n_{2}^{\prime}}\right] \leqslant 2 ;
$$

тем самым область возможных значений $\mathrm{Ch}(a)$ сужается до трех чисел: -1 , 0, 1. Наша задача - исключить два последних значения. Предположим, что $\mathrm{Ch}(a)=1$. Тогда $m=1$, и алгебра $A$ порождается двумя формами веса 1 . Поэтому $\operatorname{dim} A_{n_{1}^{\prime} n_{2}^{\prime}}=n_{1}^{\prime} n_{2}^{\prime}+1$. А по формуле для размерности

$$
\operatorname{dim} A_{n_{1}^{\prime} n_{2}^{\prime}}=1+n_{1}^{\prime} n_{2}^{\prime}+d_{1}^{\prime} n_{2}^{\prime}+d_{2}^{\prime} n_{1}^{\prime}>1+n_{1}^{\prime} n_{2}^{\prime}
$$

Противоречие.

Пусть теперь $\operatorname{Ch}(a)=0$. Тогда, прежде всего, $\operatorname{dim} A_{l} \geqslant 1$ для любого $l \geqslant 0$. В частности, $\operatorname{dim} A_{m-1} \geqslant 1$, что возможно только при $m=1$.

Далее из формулы для размерности следует, что форма $\varphi$ одинока. Если $\operatorname{deg} \psi=k>1$, то, с одной стороны, $\operatorname{dim} A_{l}=1+[l / k]$, а с другой - эта размерность равна $1+\left[l d_{1}^{\prime} / n_{1}^{\prime}\right]+\left[l d_{2}^{\prime} / n_{2}^{\prime}\right]$. Вычисляя двумя способами размерность 
пространства $A_{k}$, находим, что $\left[k d_{1}^{\prime} / n_{1}^{\prime}\right]+\left[k d_{2}^{\prime} / n_{2}^{\prime}\right]=1$. Это означает, что верно одно из двух: $k d_{1}^{\prime} \geqslant n_{1}^{\prime}, k d_{2}^{\prime}<n_{2}^{\prime}$ или $k d_{1}<n_{1}^{\prime}, k d_{2}^{\prime} \geqslant n_{2}^{\prime}$. Ввиду симметрии достаточно разобраться с первым случаем. Вычисляя двумя способами размерность пространства $A_{n_{1}^{\prime}}$, получим

$$
1+\left[\frac{n_{1}^{\prime}}{k}\right]=1+d_{1}^{\prime}+\left[\frac{n_{1}^{\prime} d_{2}^{\prime}}{n_{2}^{\prime}}\right] .
$$

По предположению $d_{1}^{\prime} \geqslant n_{1}^{\prime} / k$, и такое равенство возможно, только если $n_{1}^{\prime}=$ $k d_{1}^{\prime}$. А так как $\left(d_{1}^{\prime}, n_{1}^{\prime}\right)=1$, то $d_{1}^{\prime}=1$ и $k=n_{1}^{\prime}$. Наконец, сравним два выражения для размерности пространства $A_{n_{1}^{\prime} n_{2}^{\prime}}$. Имеем $\operatorname{dim} A_{n_{1}^{\prime} n_{2}^{\prime}}=1+n_{2}^{\prime}$ и в то же время $\operatorname{dim} A_{n_{1}^{\prime} n_{2}^{\prime}}=1+n_{2}^{\prime}+n_{1}^{\prime} d_{2}^{\prime}$ (по формуле $\left.(5)\right)$. Полученное противоречие исключает последнее нежелательное значение 0 . Предложение 2 полностью доказано.

ПреДЛОЖЕНИЕ 3. Пусть $f$ - одинокая $а$-Г-автоморфная форма. Тогда кратность $O(x)$ ее нуля в точке $x$ строго меньше порядка $\left|\Gamma_{x}\right|$ ее стабилизатора.

ДокАЗАТЕЛЬСтво. Предположим, что $O(x) \geqslant\left|\Gamma_{x}\right|$. На факторпространстве $P^{1}(\mathbb{C})$ существует мероморфная функция $\alpha$ с единственным простым полюсом в точке $\bar{x}$ (и, разумеется, с простым нулем где-то еще). Подняв $\alpha$ на верхнюю полуплоскость $H$, получим Г-инвариантную мероморфную функцию $\tilde{\alpha}(z)$ с полюсами порядка $\left|\Gamma_{x}\right|$ в точности в точках орбиты $\{\Gamma x\}$. Пусть $q=\left[O(x) /\left|\Gamma_{x}\right|\right]$. Тогда функция $f^{\prime}=f \cdot \tilde{\alpha}^{q}$ является голоморфной $a$-Г-автоморфной формой того же веса, что и $f$. Но форма $f^{\prime}$ не пропорциональна форме $f$, из чего следует, что форма $f$ не является одинокой. Полученное противоречие доказывает предложение.

СЛЕДСТВИЕ 1. Одинокая форма не может обращаться в нуль в точке с тривиальным стабилизатором. Одинокая форма не может обратиться в нуль в такой точке $x$, где $a(\gamma, x)=1$ для любого $\gamma \in \Gamma_{x}$.

ДокАЗАТЕльство. Первое утверждение следствия в доказательстве не нуждается, а второе получается так: если коцикл $a$ в точке $x$ равен 1 на стабилизаторе этой точки, то порядок нуля $O(x)$ пропорционален $\left|\Gamma_{x}\right|$ (см. п. 3.4).

Чтобы завершить доказательство необходимости, нам осталось проверить выполнение арифметических условий для коциклов первого и второго типов и всю информацию о степенях образующих и расположении их нулей. В следующем параграфе мы покажем, как это делается в наиболее сложном случае коциклов второго типа. Для них же мы докажем и достаточность условий теоремы. В оставшихся случаях доказательство как необходимости, так и достаточности, не требует привлечения новых идей и значительно проще.

\section{§6. Окончание доказательства теоремы (случай коцикла второго типа)}

Итак, нам известно, что код коцикла $a$ имеет вид $\left(-1 ; d_{1}, d_{2}, 0, \ldots, 0\right)$ (можно считать, что только $d_{1}$ и $d_{2}$ отличны от нуля) и что алгебра $A$ свободно порождается двумя формами $\varphi$ и $\psi$. Пусть $\operatorname{deg} \varphi=l_{1}, \operatorname{deg} \psi=l_{2}$ и $l_{1} \leqslant l_{2}$. Докажем, что $d_{1}^{\prime} / n_{1}^{\prime}+d_{2}^{\prime} / n_{2}^{\prime}=1+1 /\left(n_{1}^{\prime} n_{2}^{\prime}\right)$.

Для этого вычислим двумя способами размерность заведомо ненулевого пространства $A_{n l_{1}}$ (так как $\left.\varphi^{n} \in A_{n l_{1}}\right)$. По формуле (5)

$$
\operatorname{dim} A_{n l_{1}}=1-n l_{1}+\left[n l_{1} \frac{d_{1}^{\prime}}{n_{1}^{\prime}}\right]+\left[n l_{1} \frac{d_{2}^{\prime}}{n_{2}^{\prime}}\right] .
$$


$\mathrm{C}$ другой стороны, в свободной градуированной алгебре $\mathbb{C}[X, Y]$ с образующими степеней $l_{1}$ и $l_{2}$ соответственно размерность компоненты степени $n l_{1}$ равна $\left[n l_{1}^{\prime} /\left(l_{1}^{\prime} l_{2}^{\prime}\right)\right]+1=\left[n / l_{2}^{\prime}\right]+1$, где $l_{1}=s l_{1}^{\prime}, l_{2}=s l_{2}^{\prime}, s=\left(l_{1}, l_{2}\right)$. Следовательно, для любого натурального $n$ имеет место равенство

$$
\left[n \frac{l_{1} d_{1}^{\prime}}{n_{1}^{\prime}}\right]+\left[n \frac{l_{1} d_{2}^{\prime}}{n_{2}^{\prime}}\right]=\left[\frac{n}{l_{2}^{\prime}}\right]+n l_{1}
$$

или, так как $n l_{1}$ - целое число,

$$
\left[n \frac{l_{1} d_{1}^{\prime}}{n_{1}^{\prime}}\right]+\left[n \frac{l_{1} d_{2}^{\prime}}{n_{2}^{\prime}}\right]=\left[n\left(l_{1}+\frac{1}{l_{2}^{\prime}}\right)\right] .
$$

Воспользуемся теперь следующим элементарным фактом, доказанным, например, в [1].

ПРЕДЛОЖЕнИЕ 4. Если ӘЛя любого натурального $n$ выполняется равенство $[n \alpha]+[n \beta]=[n \gamma]$, то хотя бы одно из чисел $\alpha$ или $\beta$ целое и $\gamma=\alpha+\beta$.

Из этого утверждения следует, что одно из чисел $l_{1} d_{1}^{\prime} / n_{1}^{\prime}$ или $l_{1} d_{2}^{\prime} / n_{2}^{\prime}$ целое и что

$$
\frac{d_{1}^{\prime}}{n_{1}^{\prime}}+\frac{d_{2}^{\prime}}{n_{2}^{\prime}}=1+\frac{1}{l_{1} l_{2}^{\prime}}=1+\frac{1}{\left[l_{1}, l_{2}\right]} .
$$

Разумеется, замена $l_{1}$ на $l_{2}$ позволяет утверждать, что одно из чисел $l_{2} d_{1}^{\prime} / n_{1}^{\prime}$ или $l_{2} d_{2}^{\prime} / n_{2}^{\prime}$ является целым. С помощью равенства (7) мы докажем сначала взаимную простоту весов $l_{1}$ и $l_{2}$. Для этого оценим выражение $1-N+\left[N d_{1}^{\prime} / n_{1}^{\prime}\right]+$ $\left[N d_{2}^{\prime} / n_{2}^{\prime}\right]$, используя неравенство $[x]+[y] \geqslant[x+y]-1$. Имеем с учетом $(7)$

$$
1-N+\left[\frac{N d_{1}^{\prime}}{n_{1}^{\prime}}\right]+\left[\frac{N d_{2}^{\prime}}{n_{2}^{\prime}}\right] \geqslant 1-N+\left[N\left(\frac{d_{1}^{\prime}}{n_{1}^{\prime}}+\frac{d_{2}^{\prime}}{n_{2}^{\prime}}\right)\right]-1=\left[\frac{N}{\left[l_{1}, l_{2}\right]}\right] .
$$

Мы видим, что это выражение положительно для любых достаточно больших $N$. А тогда, как мы знаем (лемма 2), оно равно размерности пространства $A_{N}$, которая, таким образом, положительна для всех достаточно больших $N$. Отсюда сразу следует, что $\left(l_{1}, l_{2}\right)=1$. Здесь же отметим, что взаимная простота $l_{1}$ и $l_{2}$ гарантирует, что формы $\varphi$ и $\psi$ одиноки.

Далее, предположив для определенности, что $l_{1} d_{1}^{\prime} / n_{1}^{\prime}-$ целое число, мы покажем, что $l_{1}=n_{1}^{\prime}$, а $l_{2}=n_{2}^{\prime}$. Так как $\left(d_{1}^{\prime}, n_{1}^{\prime}\right)=1$ по условию, то $l_{1}$ делится на $n_{1}^{\prime}$. Рассмотрим выражение

$$
1-n_{1}^{\prime}+\left[\frac{n_{1}^{\prime} d_{1}^{\prime}}{n_{1}^{\prime}}\right]+\left[\frac{n_{1}^{\prime} d_{2}^{\prime}}{n_{2}^{\prime}}\right]=1-n_{1}^{\prime}+\left[n_{1}^{\prime}\left(\frac{d_{1}^{\prime}}{n_{1}^{\prime}}+\frac{d_{2}^{\prime}}{n_{2}^{\prime}}\right)\right]=1-n_{1}^{\prime}+\left[n_{1}^{\prime}+\frac{n_{1}^{\prime}}{\left[l_{1}, l_{2}\right]}\right] .
$$

Оно заведомо не меньше единицы, а это означает, что $A_{n_{1}^{\prime}} \neq\{0\}$. Но так как $n_{1}^{\prime} \leqslant l_{1} \leqslant l_{2}$, то это возможно, только если $l_{1}=n_{1}^{\prime}$. Обратимся снова к равенству (7). Оно, в частности, показывает, что $\left[n_{1}^{\prime}, n_{2}^{\prime}\right]=\left[l_{1}, l_{2}\right]$. Но мы уже доказали, что $l_{1}$ и $l_{2}$ взаимно просты, а $l_{1}=n_{1}^{\prime}$. Поэтому $n_{2}^{\prime}$ делится на $l_{2}$. Пришло время воспользоваться тем, что одно из чисел $l_{2} d_{1}^{\prime} / n_{1}^{\prime}$ или $l_{2} d_{2}^{\prime} / n_{2}^{\prime}$ является целым. Число $l_{2} d_{1}^{\prime} / n_{1}^{\prime}$ не может быть целым: это означало бы, что $l_{2}$ делится на $n_{1}^{\prime}$ и противоречило бы взаимной простоте весов. Следовательно, $l_{2} d_{2}^{\prime} / n_{2}^{\prime}$ - целое число, т. е. $l_{2}$ делится на $n_{2}^{\prime}$, и мы доказали, что $l_{2}=n_{2}^{\prime}$.

Переходим к проверке утверждения теоремы о нулях форм $\varphi$ и $\psi$. Согласно следствию 1, обе одинокие формы могут обратиться в нуль только в точках $\bar{P}_{1}$ и $\bar{P}_{2}$. Но общего нуля они иметь не могут, так как это противоречило бы 
хорошо известному факту о том, что в нашей ситуации алгебра автоморфных форм разделяет точки факторпространства [8].

Пусть форма $\varphi$ веса $n_{1}^{\prime}$ имеет нуль кратности $t$ в точке $\bar{P}_{1}$. В этом случае $t \equiv$ $n_{1}^{\prime} d_{1}\left(\bmod n_{1}\right)($ см. п. 3.4$)$, т. е. $t$ делится на $n_{1}$ (напомним, что $\left.n_{1}^{\prime}=n_{1} /\left(d_{1}, n_{1}\right)\right)$, а это запрещено одинокой форме предложением 3.

Итак, форма $\varphi$ имеет единственный нуль кратности $t_{2}$ в точке $\bar{P}_{2}$, а форма $\psi$ - единственный нуль кратности $t_{1}$ в точке $\bar{P}_{1}$. Прямое вычисление по формуле (6) (п. 3.4) показывает, что $t_{2}=\left(n_{2}, d_{2}\right)$ и $t_{1}=\left(n_{1}, d_{1}\right)$. Необходимость условий теоремы полностью доказана.

Осталось доказать, что $A$ свободна, для коцикла вида $\left(-1 ; d_{1}, d_{2}, 0, \ldots, 0\right)$ с условием $d_{1}^{\prime} / n_{1}^{\prime}+d_{2}^{\prime} / n_{2}^{\prime}=1+1 /\left(n_{1}^{\prime} n_{2}^{\prime}\right)$.

Прежде всего, формула (5) для размерности дает существование одинокой формы $\varphi$ веса $n_{1}^{\prime}$ и одинокой формы $\psi$ веса $n_{2}^{\prime}$. Далее, как и при доказательстве необходимости, пользуясь предложением 3, его следствием 1 и формулой для нулей (6), приходим к выводу, что у $\varphi$ имеется единственный нуль кратности $\left(d_{2}, n_{2}\right)$ в точке $\bar{P}_{2}$, а у $\psi-$ единственный нуль кратности $\left(d_{1}, n_{1}\right)$ в точке $\bar{P}_{1}$. С этого момента алгебраическая независимость форм $\varphi$ и $\psi$ уже не вызывает сомнений.

Далее, из формулы для размерности следует, что $\operatorname{dim} A_{n_{1}^{\prime} n_{2}^{\prime}}=2$. Ясно, что пространство $A_{n_{1}^{\prime} n_{2}^{\prime}}$ порождается формами $\varphi^{n_{2}^{\prime}}$ и $\psi^{n_{1}^{\prime}}$. Ясно также, что можно выбрать линейную комбинацию этих форм, которая обращается в нуль в произвольной точке $\bar{x} \in P^{1}$, отличной от точек $\bar{P}_{1}$ и $\bar{P}_{2}$. Соответствующую форму из пространства $A_{n_{1}^{\prime} n_{2}^{\prime}}$ обозначим через $\beta_{\bar{x}}$. Покажем, что $\beta_{\bar{x}}$ будет иметь единственный простой нуль в точке $\bar{x}$. В самом деле,

$$
\sum_{i=1}^{r} \frac{O\left(\overline{P_{i}}\right)}{n_{i}}+O(\bar{x})+\cdots=n_{1}^{\prime} n_{2}^{\prime}\left(-1+\frac{d_{1}^{\prime}}{n_{1}^{\prime}}+\frac{d_{2}^{\prime}}{n_{2}^{\prime}}\right)=1,
$$

т. е. $O(\bar{x})=1$.

Найденные формы $\varphi, \psi$ и $\beta$ уже порождают всю алгебру $A$. Убедиться в этом можно так: пусть $w-$ произвольная ненулевая форма положительного веса $N$. Предположим, что $w\left(\bar{P}_{1}\right)=0$. Тогда $O\left(\bar{P}_{1}\right) \equiv N d_{1}\left(\bmod n_{1}\right)$, т. е. кратность нуля делится на $\left(n_{1}, d_{1}\right)$. Следовательно, частное $w / \psi$ представляет собой голоморфную автоморфную форму меньшего веса. Аналогично, с помощью формы $\varphi$ можно понизить вес формы $w$ в случае, когда $w\left(\bar{P}_{2}\right)=0$. Наконец, если форма $w$ равна нулю в какой-то точке $\bar{x}$, отличной от $\bar{P}_{1}$ и $\bar{P}_{2}$, то в игру вступает форма $\beta_{\bar{x}}$, имеющая простой нуль в точке $\bar{x}$. Такая возможность понизить степень открывает дорогу индукции. Остается заметить, что формы $\beta_{\bar{x}}$ линейно выражаются через степени форм $\varphi$ и $\psi$. Теорема полностью доказана.

\section{§7. Комментарии и пример}

ПримеР. Ниже выписаны коды семи коциклов треугольной фуксовой группы $\Gamma(0 ; 2,3,7)$, приводящие к свободной алгебре $A:(1 ; 0,0,0),(0 ; 1,0,0),(0 ; 0,1,0)$, $(0 ; 0,0,1),(-1 ; 1,2,0),(-1 ; 0,1,5),(-1 ; 1,0,4)$.

ЗАмЕчАНИЕ 1. Для любых натуральных взаимно простых $n_{i}$ и $n_{j}$, больших единицы, существует единственная пара натуральных чисел $(x, y)$, таких, что $x<n_{i}, y<n_{j}$ и $x / n_{i}+y / n_{j}=1+1 /\left(n_{i} n_{j}\right)$ (алгоритм Евклида). 
ЗАмечАнИе 2 . Наша теорема верна для сигнатур $(0 ; 2,3,6),(0 ; 2,4,4),(0 ; 3,3,3)$ и $(0 ; 2,2,2,2)$ дискретных групп автоморфизмов комплексной плоскости рода 0.

ЗАмечАние 3 . Классификация $G$-пучков на $\mathbb{P}^{1}(\mathbb{C})$ со свободной алгеброй инвариантных сечений - это задача классификации (с точностью до сопряженности) линейных конечных групп комплексных отражений в $\mathbb{C}^{2}$. Она была решена Шепардом и Тоддом [12].

ЗАмЕЧАнИЕ 4. Теорема описывает и свободные алгебры инвариантов фуксовых решеток, если ограничиться коциклами $a$, которые голоморфно продолжаются в любую параболическую точку $x \in \bar{H}$ и в этой точке удовлетворяют условию $a\left(x, \Gamma_{x}\right)=1$ (или принимают в этой точке значение 1 на подгруппе конечного индекса в $\left.\Gamma_{x}\right)$.

Например, таков классический коцикл $j^{-1}$ для модулярной группы $\Gamma(0 ; 2,3, \infty)$. Код этого коцикла равен $(-1 ; 1,2,0)(-1=(-2)+1$, так как параболическая точка дает единичный вклад в число Черна). Таким образом, он является коциклом второго типа со всеми вытекающими (и хорошо известными) последствиями.

Эта работа частично была выполнена при финансовой поддержке университета г. Билефельд (Германия). Я хотел бы выразить глубокую признательность профессорам Е. Меннике и Х. Хеллингу за приглашение посетить университет летом 2000 г.

\section{ЛИТЕРАТУРА}

1. Гашков С. Б., Чубариков В. Н. Арифметика, алгоритмы, сложность вычислений. Высшая школа, М., 2000.

2. Долгачев И. В. Автоморфные формы и квазиоднородные особенности. Функц. анализ и его прил., 9, вып. 2, 67-68 (1975).

3. Кра И. Автоморфные формы и клейновы группы. Мир, М., 1975.

4. Швариман O. В. Свободные G-пучки на замкнутых римановых поверхностях. УМН, 54, вып. 6, 175-176 (1999).

5. Швариман О. В. О коциклах групп комплексных отражений и сильной односвязности факторпространств. В кн.: Вопросы теории групп и гомологической алгебры, Ярославль, 1991, с. 32-39.

6. Шимура $Г$. Введение в арифметическую теорию автоморфных функций. Мир, М., 1973.

7. Цишанг Х., Фогт Э., Колдевай Х.-Д. Поверхности и разрывные группы. Наука, М., 1988.

8. Baily W. Introductory lectures on automorphic forms. Princeton Univ. Press, 1973.

9. Dolgachev I. V. Invariant stable bundles over modular curves $X(p)$. Contemp. Math., 224, 65-69 (1999).

10. Futura M., Steer B. Seifert fibered homology 3 -spheres and the Yang-Mills equations on Riemann surfaces with marked points. Adv. Math., 96, 38-102 (1992).

11. Kawasaki T. The Riemann-Roch theorem for complex V-manifolds. Osaka J. Math, 16, 151-159 (1979).

12. Shephard G., Todd J. Finite unitary reflection groups. Canad. J. Math., 25, 159-198 (1954).

13. Wagreich $P$. Algebras of automorphic forms with few generators. Trans. Amer. Math. Soc., 262, 367-389 (1980). 